\title{
Das Bild der mährischen Slawen bei Oskar Jellinek
}

\author{
Jan Budňák
}

Der in Brünn geborene Prosaist Oskar Jellinek (1886-1949) siedelt seine Erzählungen und Novellen zumeist im ländlichen bzw. kleinstädtischen Milieu Mährens an. Obwohl er von 1904 bis 1938 in Wien gelebt hat, nach einem knappen Jahr in Brünn im April 1939 wieder fliehen musste und den Rest seines Lebens im USamerikanischen Exil verbracht hat (MUNZAR: 2002, 1), gehören seine Erzählungen eindeutig in den mährischen Kontext: Speziell in den der sog. mährischen Realisten (Ebner-Eschenbach, Saar, David), als deren eigenständiger Abschluss er mit vollem Recht betrachtet werden kann. Wir wollen versuchen uns dies vor allem anhand seiner Auffassung und Darlegung tschechischer (d. i. mährisch-slawischer) Figuren zu vergegenwärtigen.

Die Verwandtschaft von Jellineks mährischen Dorfgeschichten etwa mit denen Jakob Julius Davids liegt auf der Hand. Bereits die weibliche Hauptgestalt der ersten mährischen Novelle Jellineks („Der Bauernrichter“, 1924), die Bäuerin Wlasta Markytan, ist den Bäuerinnen Davids wie aus dem Gesicht geschnitten. Sie ist zweifelsohne nach dem Typus der schönen, kräftigen und naturnahen slawischen Frau gestaltet, was auch der (auktoriale) Erzähler selbst bestätigt: „Mit ihrem kräftigen Wuchs, der nicht ohne Höhe war, dem reichen Ährenkranz der Haare über der etwas niedrigen Stirne, der Stumpfnase über dem roten, etwas breiten Mund, der, stets halb geöffnet, gesunde Zähne zeigt, wirkte sie als der Typus der schönen slawischen Bäuerin“ (JELLINEK: 1950a, 45). Charaktertypologisch hat Wlasta Markytan also eine ähnliche physiologische Veranlagung wie die Frauen Davids; nur wird diese „Wesenhaftigkeit“ bei Jellinek zweifach „gebrochen“. Einerseits geschieht dies durch die Reflexion des Nationaltypischen in der Figur - wie aus dem obigen Zitat hervorgeht-, andererseits durch die erzwungene Abwendung der tschechischen Figur von der "nationaltypischen Substanz", wovon dann die Handlungsperipetien ausgehen. Kurz: Die urwüchsige Veranlagung der weiblichen Gestalten ist in den Novellen Jellineks wesentlich stärker gestört, in unlösbare Fragen verstrickt als bei dem etwa 30 Jahre früher schaffenden Jakob Julius David.

Die Bäuerin Wlasta Markytan muss aufgrund ihrer Ehe mit dem heimtückischen, gegenüber den kräftigen Bauern unter Minderwertigkeitskomplexen leidenden Bauernrichter“ Rafael Weynar ${ }^{1}$ ihr „Bluthaftes“2 ganz verleugnen: Der Ehemann

1 Auch er ist, trotz seines deutsch klingenden Namens, als Tschechischsprecher gekennzeichnet: Seine Frau spricht ihn mit der merkwürdigen, aber durchaus grammatischen tschechischen Vokativform „Rafku!“an.

2 Ein Ausdruck von Oskar M. Fontana, vgl. das Vorwort zu den Gesammelten Novellen Jellineks (1950). 
zwingt sie dazu. Sie darf nicht einmal mit den Bauern mitsingen: „Als der Richter das Haus betrat, begann im Dorfe gerade jener wehmütige Gesang der Bauern, den diese am Samstagabend anzustimmen pflegten, eine unendlich zärtliche, ins Hoffnungslose verschwebende Melodie. ${ }^{1}$ [...] Als sie ihren Gatten gewahrte, brach sie jäh ab. Sie mochte wissen, dass er es ungern sah, wenn sie irgendeine Fühlung mit den Bauern nahm" (JELLINEK: 1950a, 45). Ihre erzwungene Entfremdung vom einheimischen Milieu wird noch durch den Umstand erhöht, dass sie die städtische Kleidung tragen muss: Für ihre Todesszene, in der sie sich öffentlich zu ihrem Geliebten bekennt, legt sie wieder die bäuerliche an.

Ihre Pein muss im vollen Umfang ihrem Ehemann angelastet werden: Weynars Minderwertigkeitsgefühl, das ihn in die Position eines "Gescheiten“ - in Kontrast zu den kräftigen, offen handelnden Bauern - stellt, verwandelt auch sein Weib in ein Objekt seines Ehrgeizes. So nimmt es nicht Wunder, dass die junge Wlasta ein leidenschaftliches Liebesverhältnis mit dem jungen Quirin Michalek eingeht. Damit wiederholt sich die Konstellation aus den Dorfgeschichten Davids: Eine Geschichte von Liebe und Schuld um eine ursprüngliche, „nicht anders könnende“ slawische Frau. Nun findet sich dennoch ein wesentlicher Unterschied zwischen David und Jellinek: Der jüngere Autor gestaltet den Handlungsablauf noch konfliktreicher, die Figuren grübelnder, belasteter, gehemmter bei der Offenbarung aufrichtiger Beweggründe. Selbst Jurist und Richter, neigt Oskar Jellinek zur Bearbeitung von „kriminalen“ Stoffen: Die Figuren müssen oft mit einer zwingenden Determinierung fertig werden. Nur manchmal bezwingen die slawischen Protagonisten ihre Schwächen und inneren Dilemmata, sei es auch im Tode, ${ }^{2}$ manchmal gehen sie in Verzweiflung und Verwirrung unter.

Diese Umstände finden ihren Niederschlag im Bild der Tschechen, das sich aus den Novellen Jellineks ergibt. Die Tschechen Jellineks - d. i. allesamt tschechisch sprechende Mährer - tragen als ihren bestimmenden Charakterzug eine grübelnde Unheimlichkeit mit sich, eine tragische Verfangenheit im eigenen sittlichen Bewusstsein. Es ist gewissermaßen die Kehrseite der naturwüchsigen Gewissheit der Davidschen und Jellinekschen Frauen: Bei Jellinek kippt die Waage schon eindeutig um, und es rücken bei ihm Figuren in den Mittelpunkt, die an der eigenen Reinheit und Aufrichtigkeit leiden. Sei es der Bergarbeiter Mathes Benda, den seine Schuld - er hat den Liebhaber seiner untreuen Frau getötet - schließlich

1 Aus diesen Zitaten geht außer dem charaktertypischen Äußeren der slawischen Figuren ihre Musikalität hervor, die bei Jellinek - in Einklang mit David - ein fester Bestandteil des mährischslawischen Dorfmilieus ist.

2 Dies sei kurz anhand des Ausgangs der Novelle „Der Bauernrichter“ angedeutet: Die ständig gedemütigte Wlasta offenbart ihre außereheliche Liebe zu Quirin öffentlich, wodurch sie diesem das Leben rettet. Nach dem Bekenntnis wird sie jedoch von ihrem Ehemann, dem hinterlistigen Bauernrichter, getötet. Dieser wird zwar sogleich verhaftet, überwindet jedoch gerade dadurch die Quelle seiner Minderwertigkeitsgefühle gegenüber den Bauern: „Er hatte kraftvoll gehandelt wie ein Bauer" (S. 66). 
doch einholt, sei es der Offizierskoch Valnocha (JELLINEK: 1950c), ${ }^{1}$ der einfach nicht anders kann, als alle Offiziere - ironischerweise außer dem Verführer seiner Verlobten - mit Hilfe von giftigen Pilzen ins Jenseits zu befördern. Hier können wir schlussfolgern, dass die mährisch-slawischen Gestalten Jellineks eine Brücke schlagen zwischen den naturwüchsigen Dorftypen der realistischen Erzähler und denjenigen slawischen Typen, die im Werk der mährischen Expressionisten agieren (vgl. die mährischen Novellen Hermann Ungars „Ein Mann und eine Magd“ und „Geschichte eines Mordes“ bzw. die von Ernst Weiß „Stern der Dämonen“ und „Franta Zlin“). Die Wendung ins Maßlose bzw. Ungeheure, die bei Weiß oder Ungar oft mit den (mährischen) Slawen verknüpft ist, taucht nicht erst bei Jellineks leidenden Aufrichtigen auf; sie ist genau genommen bereits bei Davids naturnahen Frauen vorhanden.

Jellinek benutzt eine eigene Art und Weise, das Milieu, über das er schreibt, als tschechisch zu kennzeichnen. Nachdem, was wir bereits oben über das Werk von Jellinek gesagt haben, ist kaum zu erwarten, dass der Autor explizite psychologisierende Schlussfolgerungen aufgrund der Nationalität seiner Figuren ziehen wird. Zweifellos weiß er doch um die Existenz eines slawischen Typus im Bezug auf Körperbau bzw. auf das Seelische: „Und die Backenknochen des slawisch geschnittenen Gesichtes, mit der breiten, flach aufliegenden Nase, sprangen seit der Haft noch schärfer hervor als bis dahin," (JELLINEK: 1950b, 297) lautet die äußere Charakterisierung der Titelgestalt von Jellineks letzter Novelle, „Der Freigesprochene“. Auch hier ist „das Slawische“ keine psychologische Determinante, sondern eine Begleiterscheinung des inneren Zustands. Wir merken den beiden „slawischen“ Aussagen Jellineks eindeutig Distanz an: Das Gesicht von Wlasta ist lediglich "slawisch geschnitten“ [Hervorhebung J. B.], sie wirkt lediglich „wie der Typus“ der slawischen Bäuerin, ohne das damit etwas über ihre „nationalen Charaktereigenschaften“ gesagt wäre.

Eine Art erbliche nationale Veranlagung lassen die überaus objektiven, neutralen Erzähler Jellineks trotzdem gelten: „'Tatinku! Tatinku!' so rief der Kleine ihm [seinem Vater Mathes Benda, J. B.] nun immer entgegen, sobald er die Stube des Ambrož betrat, wo er jetzt die Abende verbrachte, wenn er nicht bei dem bedenksamen Habrman saß, der, wie er selbst, von einem schlesischen Urgroßvater deutsches Blut in den Adern hatte und ihm nach dem Ambrož der Liebste war" (JELLINEK: 1950b, 336). Da Mathes Benda - die Titelgestalt - auch selbst durchaus „bedenksam“, ja gefährlich „kopflastig“ ist, lässt sich diesem unauffälligen Hinweis auf das „deutsche Blut“ ziemlich große Bedeutung beimessen: Hier wird, wenn auch unscheinbar, so doch klar erkennbar aus dem „nationalcharakterlichen“ Ideengut

1 Wie wichtig das Schicksal des einfachen, aber eben nicht anders könnenden Mannes für den Verfasser ist, können wir aus einem seiner Gedichte schlussfolgern. Jellinek bezeichnet sich selbst als „den Historiker Valnochas“: „Was namenlos hin durch das Dasein wallt,/Dem gebe ich Namen und geb' ihm Gestalt:/ [...] Ich präge, wen Gott zu prägen vergaß,/Ich bin der Historiker Valnochas“ (Vorwort, S. 16f.). 
geschöpft. Es gilt jedoch nach wie vor, dass in Jellineks Prosa solche Äußerungen ganz und gar vereinzelt sind. So wage ich zu behaupten, dass die verhältnismäßig sinnlichen Figuren Minka (die untreue Frau Bendas) und Petr Ambrož aus der Erzählung nicht als markiert slawische herausstechen gegenüber den "Grüblern“ Benda und Habrman, die von ihren schlesischen Vorfahren „deutsches Blut" geerbt haben. Im Gegenteil: Die Männer, standfeste und gerechte Bergarbeiter, werden über nationale Grenzen hinweg als eine einheitliche Gruppe dargestellt, vor allem in Bezug auf ihre sittlichen Lebensgrundsätze und ihre Hilfsbereitschaft. Religiöse und erst recht nationale Fragen spielen für sie keine Rolle: Auch der strenge aber gerechte Buchbinder Jan Šmid, der den von Schuldgefühlen gepeinigten „Freigesprochenen“ erst wirklich freispricht, bindet in seiner Werkstatt sowohl deutsche als auch slawische Bücher (JELLINEK: 1950b, 330).

Trotzdem werden wir dauernd daran erinnert, dass wir es mit slawischen Figuren zu tun haben. Dies bewerkstelligt der Erzähler nicht nur durch nationalpsychologisierende Rückschlüsse, sondern auch durch die konsequente Benutzung von tschechischen Ausdrücken in der Figurenrede. Die Regel ist dies in Anreden: „'Also, sie verstehen, lieber pane Benda!' [...] Also, beehren $S^{\prime}$ mich halt bald wieder - ich mein': auf gemütlicher ... Ma ucta, pane Benda, ma ucta!' [...] 'Ano, pane Šmid, ich versteh' Sie sehr gut. '“ (JELLINEK: 1950b, 329ff.) Obgleich man schon diesen Zitaten entnehmen kann, dass Jellinek zumindest die tschechische Orthographie nicht einwandfrei beherrscht, verfügt er doch über einige gängige tschechische Ausdrücke und Wendungen. Diese ziehen im neutral, fast perspektivlos gehaltenen Erzählfluss der Novellen ${ }^{1}$ den Eindruck des tschechischen Milieus nahezu mit Selbstverständlichkeit nach sich: Es werden tschechische Vokativformen gebildet, tschechische Diminutive benutzt (Fanuschka, Mathitschek), tschechische Ausrufe den Figuren in den Mund gelegt („Ježiž - Maria - und Josef!“, „to je leš, leš "), und manchmal auch Längeres, das dann jedoch bedeutendere Mängel in der Beherrschung des Tschechischen erkennen lässt: „Pantato ... je dobr, velmi dobr ... no, Pantato“ (JELLINEK: 1950b, 335). ${ }^{2}$ Das tschechische Milieu wird also objektiver, „äußerlicher" als bei den anderen mährischen Realisten kenntlich gemacht. Die Nationalität trägt jedoch nichts Wesentliches zur Psychologie der Figuren bei.

Es wäre gewagt, eine genau umrissene Brücke zwischen Jellineks Stoffen, die sich mit lebenswichtigen ethischen Überzeugungen bzw. Konflikten befassen, und ihren slawisch-mährischen Verkörperungen schlagen zu wollen. Nichtsdestotrotz wurde in mehreren ausgezeichneten Prosatexten vom Autor selbst diese Brücke geschlagen,

1 Jellineks Erzählungen sind eigentlich keine klassischen Novellen. Sie haben weder die RahmenBinnen-Struktur, noch die Perspektivierung der einzelnen Teile durch ihre jeweiligen Erzähler. Da jedoch selbst die „kanonische“ Ausgabe von Jellineks Prosa im Zsolnay Verlag den Titel Gesammelte Novellen führt, ist da wohl nichts zu machen.

2 Auf Deutsch würde die Passage etwa lauten: „Herr Vater, ... es ist gut, sehr gut“. In korrektem Tschechisch müsste es heißen: „Pantáto ... to je dobře, velmi dobře“. 
ohne groß und breit kommentiert zu werden: Die mährischen Dorfbewohner bzw. die abgehärteten Bergarbeiter hielt Jellinek wohl diesbezüglich für geeignet. Auch in dieser figurentypologischen Linie, wie sie klar genug aus der Komparation des Tschechenbildes der mährischen Realisten hervorgeht, passt also Oskar Jellinek in den mährischen Zusammenhang: Auch ihm sind die mährischen Dorfbewohner ein würdiger Träger tiefer moralischer Botschaften, die dennoch nicht auf ihre von den Autoren unterschiedliche Nationalität zurückgeführt werden, sondern selbst Sinn und Zweck der literarischen Betrachtung sind.

\section{Zusammenfassung:}

In diesem Beitrag werden die slawischen Figuren des in Brünn geborenen Prosaisten Oskar Jellinek unter dem Aspekt ihrer Charakterzüge betrachtet und der Versuch einer Einordnung der Prosawerke Jellineks in das breitere Umfeld der sog. mährischen Realisten (Marie von Ebner-Eschenbach, Ferdinand von Saar, Jakob Julius David) unternommen. Es wird dabei nicht nur vornehmlich die Physiognomie der untersuchten Figuren in Betracht gezogen, sondern auch eine Art tschechische (d. h. mährische) „Physiognomie“ der Landschaft, die Jellinek in seinen Werken als ein tschechisches Charakterisitikum zu veranschaulichen sucht.

Schlüsselwörter:

Oskar Jellinek - mährische Realisten - Figurenanalyse - Prosawerke

\section{Literatur:}

JELLINEK (1950a): Jellinek, Oskar. Der Bauernrichter, in: ders.: Gesammelte Novellen. Wien: Paul Zsolnay, 1950, 29-66.

JELLINEK (1950b): Jellinek, Oskar. Der Freigesprochene, in: ders.: Gesammelte Novellen. Wien: Paul Zsolnay, 1950, 293-378.

JELLINEK (1950c): Jellinek, Oskar. Valnocha, der Koch, in: ders.: Gesammelte Novellen. Wien: Paul Zsolnay, 1950, 143-193.

MUNZAR (2002): Munzar, Jiří. Oskar Jellinek, in: Fiala-Fürst, Ingeborg; Krappmann, Jörg (Hg.). Lexikon deutschmährischer Autoren. Olomouc: Univerzita Palackého, 2002. 